\title{
Coverillustration
}

\section{My, what big eyes you have... .}

The Jurassic landscape of 160 million years ago must have been a fearsome and unforgiving habitat with terrestrial tetrapods fearing such creatures as Dilophosaurus among other dramatic predators. The seas of that era contained a group of ferocious predators not yet popularised by movies or toys, or even in our common lexicon, but contemporaries of the dinosaurs - the ichthyosaurs. These creatures were highly successful and represent a dynasty of the Jurassic seas for perhaps 150 million years from 245-65 million years ago. Some of the larger of these ichthyosaurs could have had Dilophosaurus for lunch if the latter were foolhardy enough to enter the shallow seas.

Larger and longer than a London double decker bus, Temnodontosaurus or "cutting tooth lizard" successfully ruled the seas, red in tooth and fin, and was among the largest aquatic predators ever, perhaps with the exception Carcharodon megalodon, although the two probably never met.

To fill the niche of deeper water, however, these ichthyosaurs had to develop the visual resources to address these low light habitats. The visual imperative of feeding at 500-1000 metres or more, instead of the shallow seas of the continental shelf, would have required the development of large eyes with a surprising ability to gather light.

Although the ichthyosaurs (and the much later appearing Carcharodon megalodon) are long extinct, there is much to learn from these spectacular creatures. With eyes larger than footballs, ichthyosaurs had the largest eyes ever known, and we know much of their lifestyle and habits.

Although Temnodontosaurus was the second or third largest known ichthyosaur but had the largest known eyes ever, our cover features an ichthyosaur (Ophthalmosaurus icenicus) that was perhaps more versatile and interesting. O icenicus was another species of parvipelvian ichthyosaur with very large eyes, only slightly smaller than the eyes of Temnodontosaurus, but $O$ icenicus was less than half the size, and much more manoeuvrable than Temnodontosaurus. Ophthalmosaurus, or "eye lizard," has the distinction of having the largest ocular diameter compared with the body length of any animal, ever. Resembling the morphology of a modern dolphin, $O$ icenicus was only slightly over 4 metres long and weighed approximately $950 \mathrm{~kg}$, the head being $20 \%$ of that length (Motani R, Sci Am 2000;283:52-9). The young were born alive and were probably immediately brought to the surface by the mother or instinctively sought the surface as other pelagic birthing marine mammals do today. These air breathing predators evolved from terrestrial ancestors, who themselves had evolved from fish.

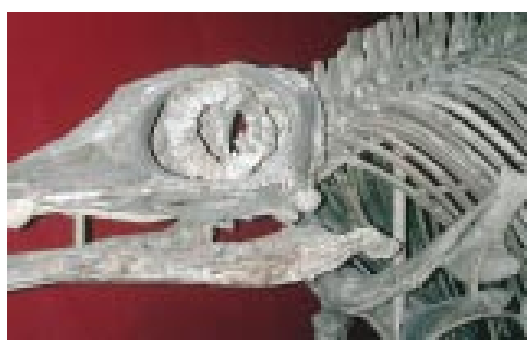

Ophthalmosaurus had a long thin mandible that would have been an excellent tool for capturing fast, manoeuvrable prey such as large fish and squid, its principal diet. This sleek ichthyosaur was probably active at night, a deep diving species, or both, allowing it to fill a rather specialised marine niche. The animal possessed a cartilaginous upper tail fin which initially puzzled paleontologists because the cartilage was not preserved in the fossil record, and the tail appeared to bend sharply downward without an upper fin. This misunderstanding was corrected when an impression of the entire fin revealed an upper fin supported by cartilage. The ample fossil record provides much information and allows for understanding of the size, and to some extent, the function of the eyes. The size of the eyes can be determined by the fossilised sclerotic plates or sclerotic rings that are clearly visible on the cover photograph. These sclerotic plates are seen in many other vertebrates, although not in humans. Birds, especially, have these sclerotic plates and they serve several functions; they help to stabilise globes that are asymmetric, and probably also helped to support the large eyes in these ichthyosaurs against the extreme pressure of oceanic depths of 1000 metres of more. As can be seen in at least some birds, these sclerotic plates may even be capable of marrow production. These plates overlap and serve as reinforcement for the anterior segment of the globe that would have been externally exposed, creating an almost complete bony chamber.

Perhaps the most prized information garnered from the fossil record includes the eye size and capabilities. The sclerotic plates allow for the calculation of the diameter of these eyes, and the largest of these has been found to be $264 \mathrm{~mm}$ in diameter in a specimen of Temnodontosaurus platyodon. Ophthalmosaurus had eyes only slightly smaller and some have been found that are $230 \mathrm{~mm}$ in diameter, but this ichthyosaur had a much smaller body and weighed far less.

More information can be determined from these fossil records. The f-number of the eye can be approximated with this information. The f-number is a method of measuring the relative light gathering abilities of the eye- or of a camera lens, for that matter. The smaller the f-number, the more light the system can absorb and utilise. A human eye has an f-number of approximately 2.1, some species of owls approximately 1.1, and Ophthalmosaurus from 0.8 to 1.1. A large eye such as the one possessed by $O$ icenicus with an f-number of 0.9 would have been able to perform well in an extraordinarily dim environment. An optical instrument that large, with an f-number that small, would be an expensive lens system indeed.

Evolutionarily, paleontologists now believe that ichthyosaurs probably branched off the diapsids (two pairs of openings in the skull in addition to the eyes), at about the time lepidosaurs, which includes snakes and lizards, and archosaurs, which includes the crocodiles, birds, and dinosaurs, diverged. These were a distinct group and more closely related to reptiles than the dinosaurs. The enormous eyes of Ophthalmosaurus, though, were never repeated, at least in the vertebrate world, although an invertebrate, the giant squid, is still extant and has a similar sized eye at $250 \mathrm{~mm}$. The giant squid is a much larger animal than Ophthalmosaurus, yet probably feeds at similar or even deeper depths than $O$ icenicus.

Ivan R Schwab

University of California, Davis, 4860 Y St, Suite 2400, Sacramento, CA 95817 , USA: irschwab@ucdavis.edu

Thanks to the Natural History Museum (London) for allowing the photography. (OThe Natural History Museum. Thanks to Ryosuke Motani, PhD, for his suggestions and cover inspiration. Thanks to Santen, Inc, for the sponsorship of the cover image. 